\title{
ABUNDÂNCIA E FENOLOGIA DE CRYPTOCELLUS SIMONIS HANSEN \& SØRENSEN, 1904 (RICINULEI, ARACHNIDA) NA SERAPILHEIRA DO BOSQUE RODRIGUES ALVES, BELÉM, PARÁ, BRASIL, COM A COMPARAÇÃO DE TRÊS TÉCNICAS DE COLETA
}

\author{
José Augusto Pereira Barreiros', Ricardo Pinto-da-Rocha² \& Alexandre Bragio Bonaldo ${ }^{3}$
}

Biota Neotropica v5 (n1a) - http://www.biotaneotropica.org.br/v5n1a/pt/abstract?article+BN004051a2005

\begin{abstract}
Recebido em 07/12/2003
Aceito em 01/02/2005

${ }^{1}$ Programa de Pós-Graduação em Zoologia, Museu Paraense Emílio Goeldi / Universidade Federal do Pará, Caixa Postal 399, CEP 66040-170, Belém, PA, Brasil, Bolsista CAPES - Mestrado. E-mail: jbarreiros@museu-goeldi.br

${ }^{2}$ Departamento de Zoologia, Instituto de Biociências, Universidade de São Paulo, Caixa Postal 11461, CEP 05422-970, São Paulo, SP, Brasil E-mail: ricrocha@usp.br

${ }^{3}$ Departamento de Zoologia, Museu Paraense Emílio Goeldi, Caixa Postal 399, CEP 66040-170, Belém, PA, Brasil. E-mail:bonaldo@museu-goeldi.br
\end{abstract}

\begin{abstract}
The abundance and phenology of a population of Cryptocellus simonis from Rodrigues Alves Park, Belém, Pará State, Brazil was studied. A total of 159 concentrated litter samples (each one with $1 \mathrm{~m}^{2}$ ) were obtained, 53 manually sorted, 53 processed in a Berlese-Tullgren apparatus and 53 processed in a Winkler apparatus. These three techniques produced 111 individuals (11 males, 15 females, 61 nymphs and 24 larvae). The median and highest densities were 0.7 and 18 ind./m², respectively. During the rainy season (March to May) the juveniles represented $78 \%$ of all collected individuals (eight larvae, 16 protonymphs, seven deutonymphs, eight tritonymphs and 11 adults), while during the dry season (September to November) the juveniles represented $75 \%$ of the total catch (16 larvae, nine protonymphs, 11 deutonymphs, 10 tritonymphs and 15 adults). No significant differences in the abundance of $C$. simonis were detected comparing the rainy and dry seasons. The development stages showed a significant positive correlation between the carapace and femur III lengths. Winkler bags represented $63 \%$ of all catches and were the best collecting technique for ricinuleid sampling.
\end{abstract}

Key words: Cryptocellus simonis, Ricinulei, abundância, fenologia, Amazônia Oriental.

\section{Resumo}

Estudou-se a abundância e a fenologia de uma população de Cryptocellus simonis, no Bosque Rodrigues Alves, região metropolitana de Belém. Foram coletadas 159 amostras de $1 \mathrm{~m}^{2}$ de serapilheira concentrada, 53 processadas manualmente, 53 em funis de Berlese-Tullgren e 53 com extratores de Winkler. Obteve-se um total de 111 indivíduos, sendo 11 machos, 15 fêmeas, 61 ninfas e 24 larvas. As densidades média e máxima foram 0,7 e 18 ind./m², respectivamente. No período chuvoso (março a maio), os jovens representaram 78\% do total de indivíduos (oito larvas, 16 protoninfas, sete deutoninfas, oito tritoninfas e 11 adultos). No período seco (setembro a novembro), os jovens corresponderam a $75 \%$ (16 larvas, nove protoninfas, 11 deutoninfas, 10 tritoninfas e 15 adultos). Não foram encontradas diferenças significativas na abundância de $C$. simonis entre os períodos seco e chuvoso, porém foi encontrada correlação positiva e significativa entre o comprimento da carapaça e o comprimento do fêmur III, em cada estágio de desenvolvimento. O extrator de Winkler representou 63\% do total de indivíduos e foi a melhor técnica na captura de Ricinulei.

Palavras-chave:Cryptocellus simonis, Ricinulei, abundância, fenologia, Amazônia Oriental. 


\section{INTRODUÇÃO}

A ordem Ricinulei inclui aracnídeos que compartilham uma estrutura em forma de capuz (cúculo) que recobre as quelíceras (Platnick 2002) e as fêmeas carregam um único ovo (Legg 1977). Uma das características mais peculiares deste grupo é o modo de transferência de espermatozóides, realizado através de um elaborado aparato copulatório localizado na terceira perna do macho, cujos caracteres são, em geral, espécie-específicos (Harvey 2002). No passado, os Ricinulei foram considerados extremamente raros. No entanto, estudos realizados nas florestas da Amazônia Central indicam que estes animais, apesar de ainda serem considerados raros em relação a outros artrópodes de solo, podem ser muito mais abundantes em certas áreas do que se imaginava (Platnick 1988; Adis et al. 1989). Entretanto, alguns levantamentos extensivos da fauna de solo na Amazônia Oriental não relataram a ocorrência destes animais (Bandeira \& Torres 1985, 1988, Macambira 1997). Segundo Adis \& Harvey (2000) e Platnick (2002), a Ordem Ricinulei apresenta apenas uma família recente, Ricinoididae, composta por três gêneros: Ricinoides (com apenas espécies africanas), Pseudocellus (com espécies neotropicais e neárticas) e Cryptocellus (com espécies exclusivamente Neotropicais). Apenas cinco espécies de Cryptocellus são encontradas na Amazônia Brasileira: C. foedus Westwood (localidade não especificada), C. simonis Hansen \& Sørensen (estado do Pará), C. becki Platnick \& Shadab (estado do Amazonas), C. adisi Platnick (também do Amazonas) e $C$. abaporu Bonaldo \& Pinto-da-Rocha (estado de Rondônia) (Platnick \& Shadab 1977; Platnick 1988; Bonaldo \& Pintoda-Rocha 2003).

De acordo com Adis et al. (1989) os Ricinulei tropicais são encontrados no solo e na serapilheira de florestas nãoinundáveis. Podem também ser encontrados em cavernas e até mesmo em áreas perturbadas, como plantações de café (Platnick 2002). São predadores de pequenos insetos imaturos e aracnídeos jovens (Platnick 2002). Poucos são os trabalhos que mencionam dados ecológicos, como densidade de algumas espécies (Platnick \& Pass 1982). Os únicos trabalhos existentes sobre ecologia de Ricinulei para a Amazônia são os de Adis et al. (1989) e Adis et al. (1999), que abordaram populações de Cryptocellus becki e de $C$. adisi nos arredores de Manaus, Amazonas. Platnick \& Paz (1979) destacaram a escassez de espécimes depositados em coleções, sendo a maioria das espécies descritas conhecidas por apenas poucos exemplares. Segundo Harvey (2002) as espécies de Ricinulei apresentam uma distribuição limitada, sendo que muitas delas são encontradas em apenas uma localidade. Este autor chamou a atenção para os riscos de extinção que este grupo sofre devido ao desmatamento e a alteração das florestas primárias pela ação do homem. Isto é extremamente notável, para algumas áreas da floresta Amazônica, especialmente as próximas aos grandes rios e estradas. De acordo com Overal \& Mascarenhas (1993), o levantamento da fauna Amazônica prossegue tão lento que muitas espécies podem até desaparecer antes de serem conhecidas pela ciência, sendo desconhecidos os impactos decorrentes de atividades econômicas e da ocupação humana na Amazônia. Estes autores indicam que as áreas transformadas ou ocupadas pelo homem também devem ser incluídas no inventário biológico. A região metropolitana de Belém carece ainda de um estudo quali-quantitativo da fauna de Ricinulei remanescente nos ambientes urbanos e nas matas do entorno da cidade. O inventário faunístico e a quantificação da biodiversidade destas áreas são fundamentais para o entendimento, em longo prazo, dos impactos ambientais da urbanização sobre as florestas tropicais.

Cryptocellus simonis Hansen \& Sørensen foi descrita com base em um único espécime coletado na localidade "Le Pará” (Belém do Pará, segundo Levi 1964). A revisão de Platnick \& Shadab (1977) registrou outros dois indivíduos, coletados na Mata do Utinga (região metropolitana de Belém). O presente estudo aborda resultados do primeiro levantamento da macrofauna de aracnídeos de serapilheira do Bosque Rodrigues Alves, Belém. Esta área sofre com a pressão da expansão urbana desde o início do século XX, e atualmente é uma ilha de mata cercada por uma área totalmente urbanizada. Os dados fenológicos desta população, comparando a eficiência de três técnicas de coleta de artrópodes de solo são apresentados. Esta comparação visa otimizar a amostragem de Ricinulei em situações de inventários faunísticos.

\section{MATERIAL E MÉTODOS}

\section{1 Área de estudo}

O Bosque Rodrigues Alves encontra-se localizado em meio ao espaço urbano da cidade de Belém, no estado do Pará, Brasil (1²5’49’'S, 48²7’22”'O). Possui uma área de 15 ha $\left(150.000 \mathrm{~m}^{2}\right)$ constituída por um resquício de floresta primária de terra firme que abriga espécies da fauna e flora amazônica (figura 1). Este espaço foi constituído em 1883 com o objetivo de ser uma importante reserva natural de espécies vegetais da floresta de terra firme do estuário Amazônico, constituindo uma alternativa de lazer à população. No início do século XX, muitas árvores foram eliminadas para dar lugar a trilhas, viveiros de animais, lagos, canais e pequenas construções. Houve também a introdução de algumas plantas exóticas. Entretanto, a maior parte da vegetação é original. Atualmente, o Bosque Rodrigues Alves representa uma ilha de mata retangular, cercada por áreas urbanizadas e dividido em um mosaico de canteiros de tamanhos variados e diferentes graus de antropização. As maiores árvores ultrapassam $40 \mathrm{~m}$ de altura, contudo a maioria do dossel é composta por árvores com cerca de 30 m (F. A. Contente, comunicação pessoal). 


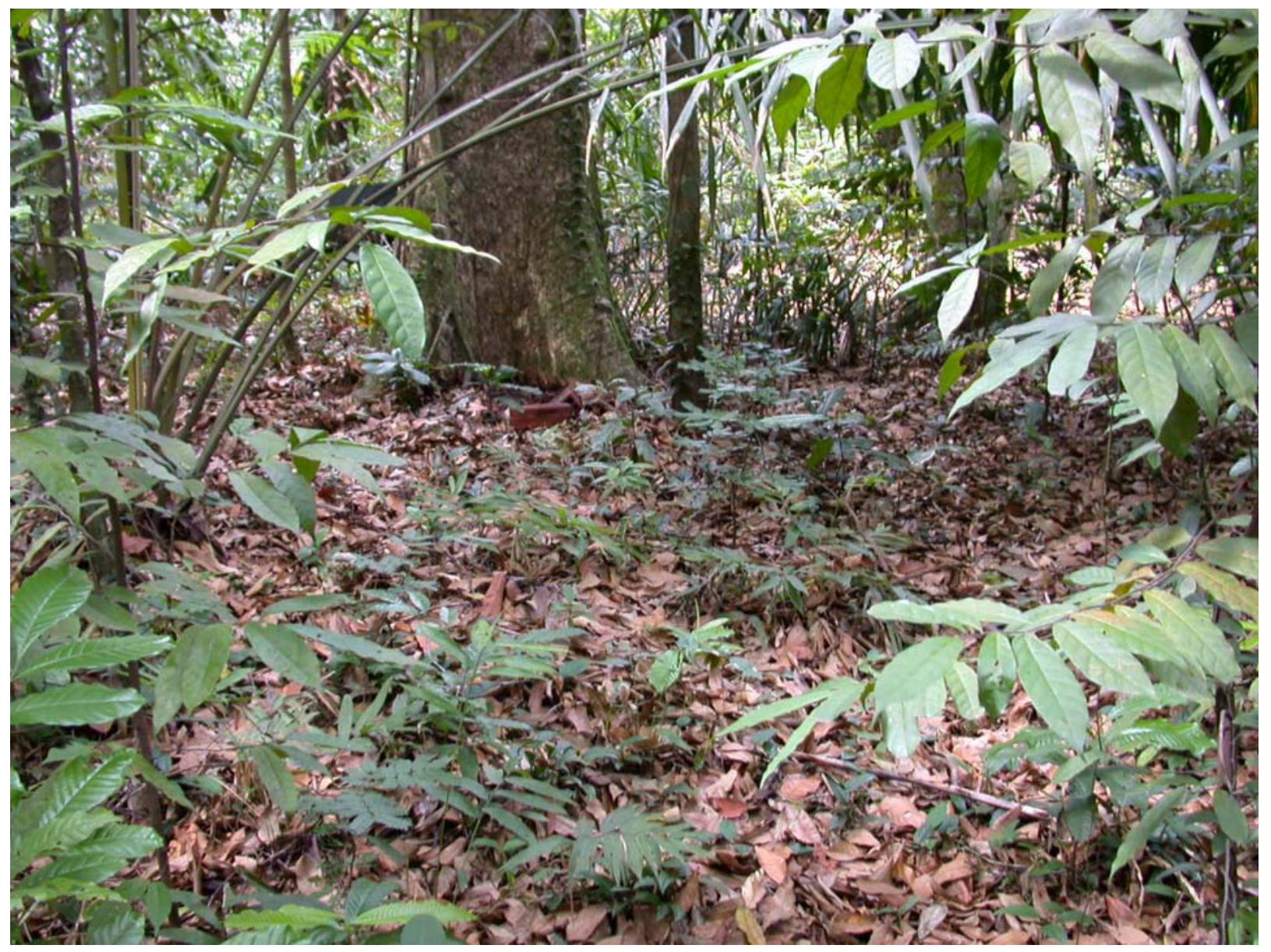

Figura 1. Aspecto da mata de terra firme do Bosque Rodrigues Alves, Belém, Pará, durante o período seco. Foto: R. Pinto-da-Rocha.

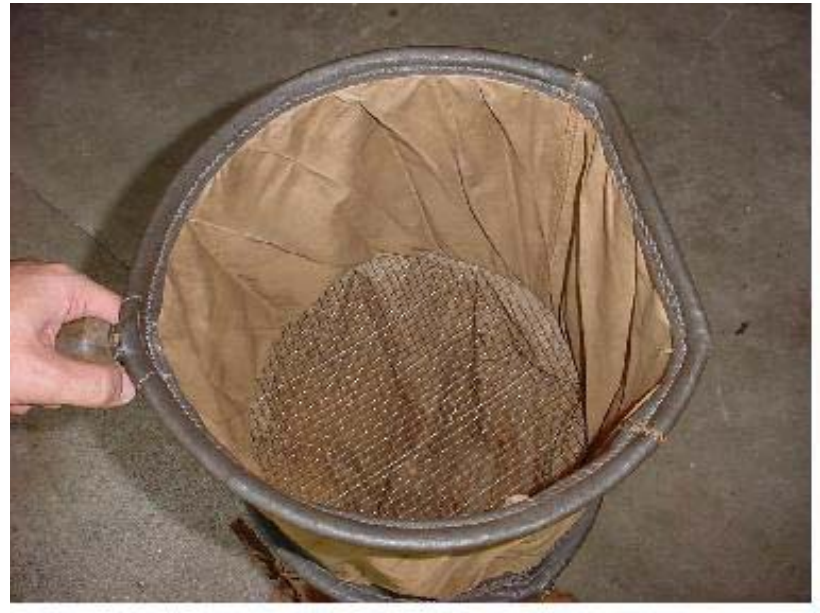

Figura 2. Peneira utilizada como concentrador de serapilheira. Foto: A. L. Prudente.

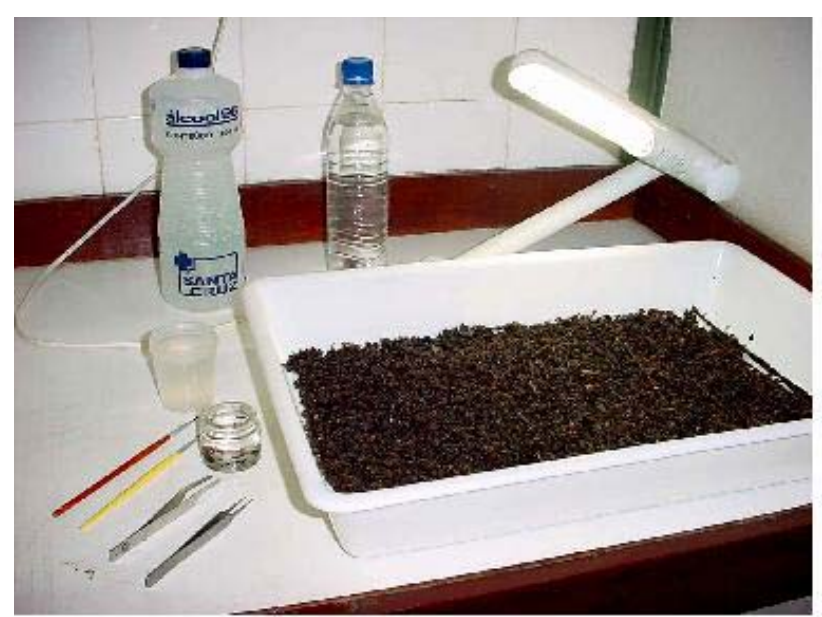

Figura 3. Bandeja utilizada na triagem manual de serapilheira no Laboratório de Aracnologia do Museu Paraense Emílio Goeldi. Foto: A. L. Prudente. 

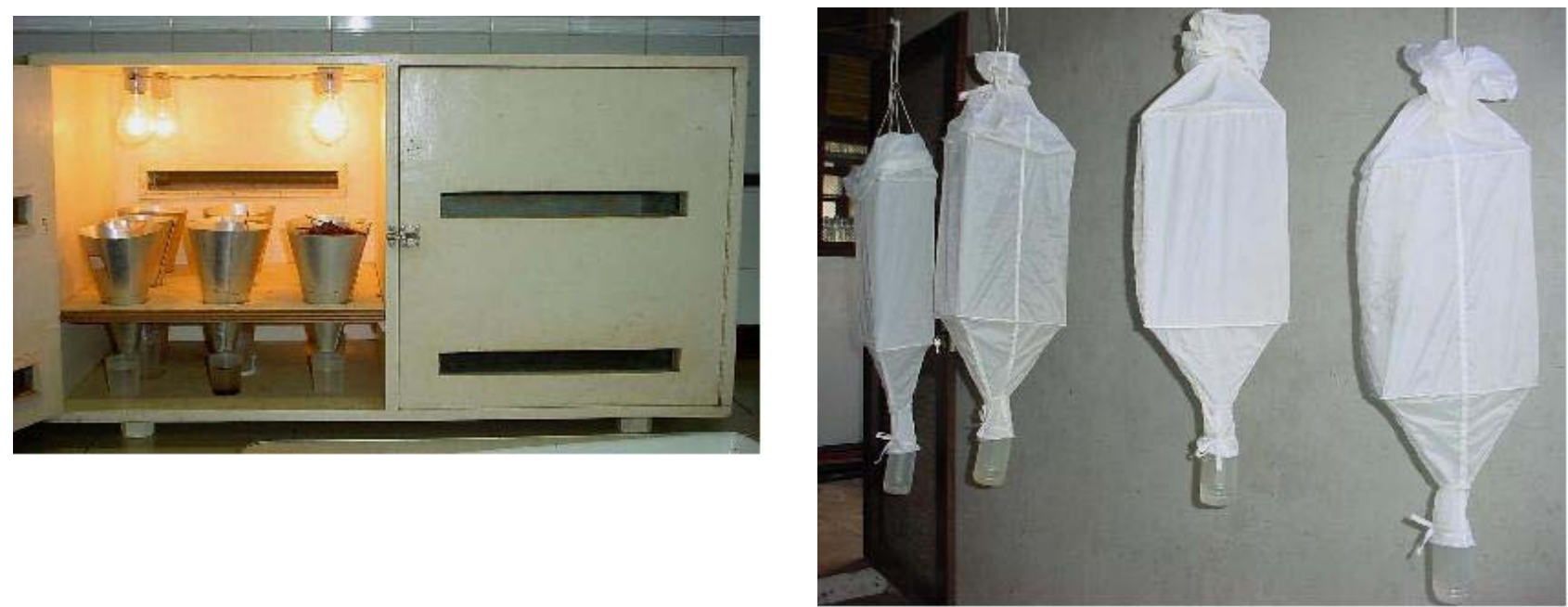

Figura 4. Funil de Berlese-Tullgren no Laboratório de Aracnologia do Museu Paraense Emílio Goeldi. Foto: A. L. Prudente.
Figura 5. Extrator de Winkler montado no Laboratório de Aracnologia do Museu Paraense Emílio Goeldi. Foto: A. L. Prudente.

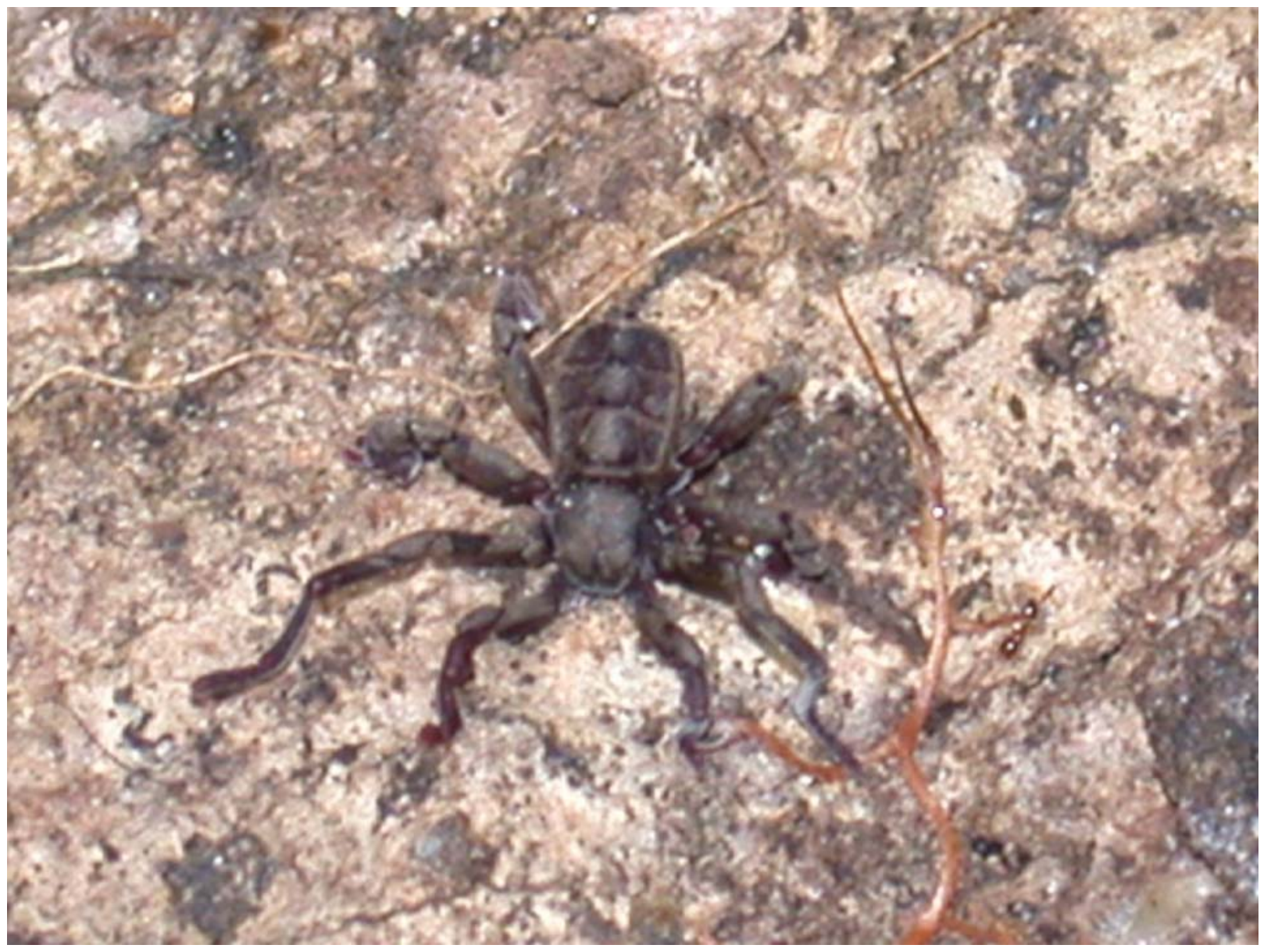

Figura 6. Adulto de Crytocellus simonis (macho) na serapilheira do Bosque Rodrigues Alves. Foto: R. Pinto-da-Rocha. 


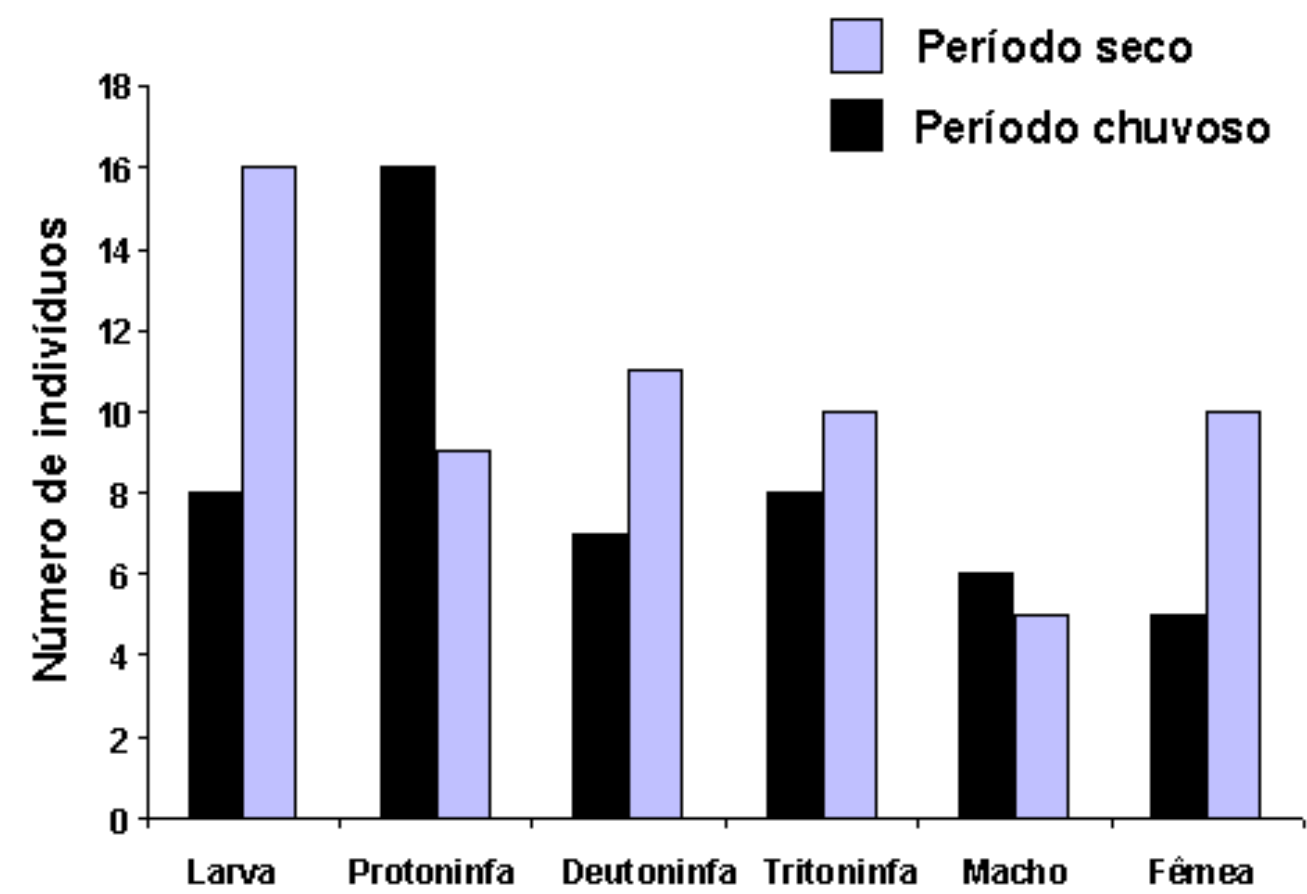

Figura 7. Abundância de Cryptocellus simonis, por estágio de desenvolvimento, na serapilheira do Bosque Rodrigues Alves, Belém, Pará, Brasil, durante os períodos seco e chuvoso.

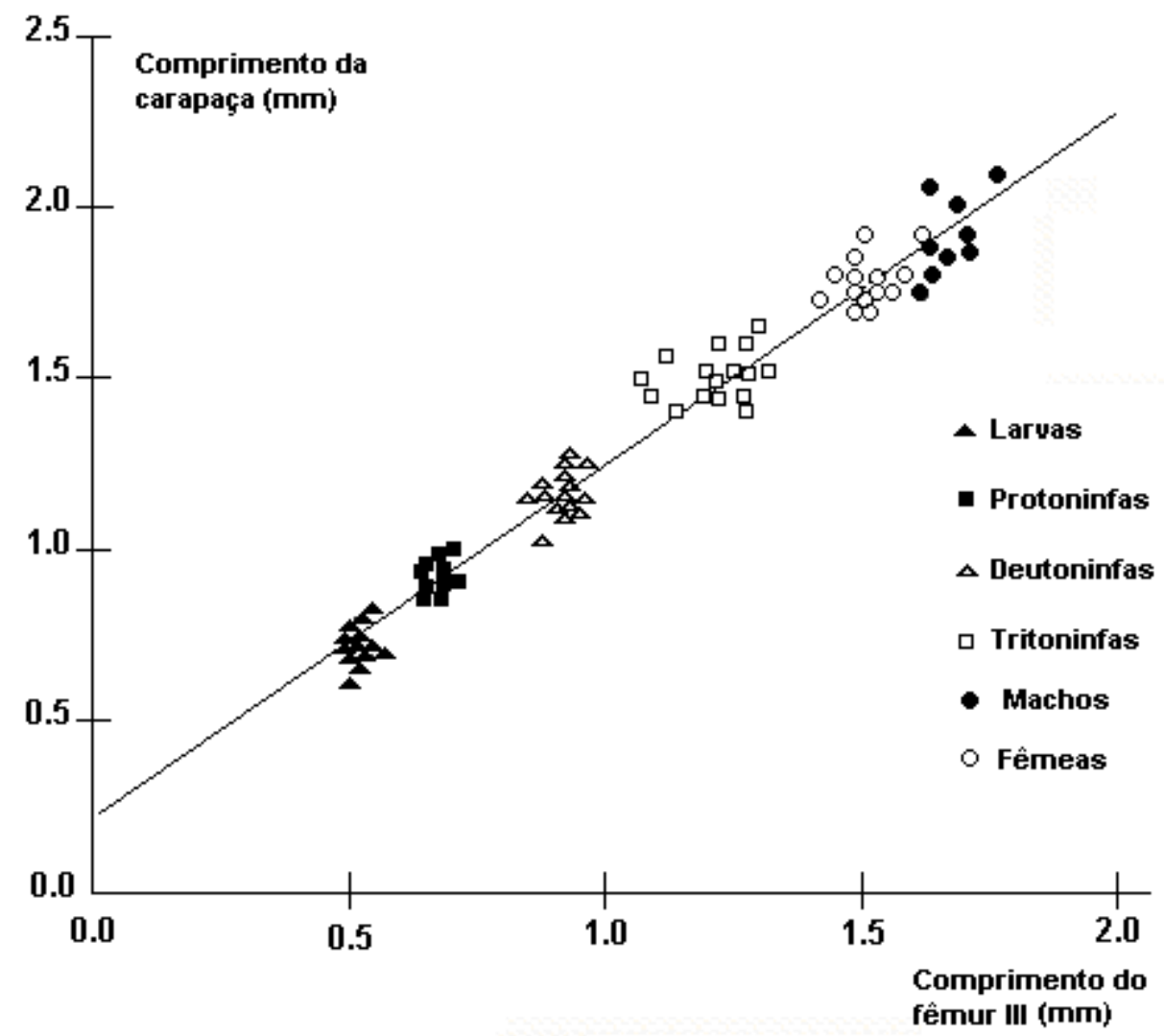

Figura 8. Relação entre o comprimento da carapaça e o comprimento do fêmur III dos estágios de desenvolvimento de Cryptocellus simonis na serapilheira do Bosque Rodrigues Alves, Belém, Pará, Brasil coletados entre março-novembro de 2001 (Correlação Linear de Pearson, $r=0,9874, p=0,00001)$. 


\begin{tabular}{ccccc}
\hline $\begin{array}{c}\text { Estágio de } \\
\text { desenvolvimento }\end{array}$ & $\begin{array}{c}\text { Comprimento da carapaça }(\mathrm{mm}) \\
\text { Média }\end{array}$ & $\begin{array}{c}\text { Comprimento do fêmur III (mm) } \\
\text { Desvio Padrão }\end{array}$ & Média & Desvio Padrão \\
\hline Larva & 0,7175 & 0,0437 & 0,5183 & 0,0201 \\
Protoninfa & 0,916 & 0,0439 & 0,6584 & 0,0316 \\
Deutoninfa & 1,1744 & 0,0633 & 0,9117 & 0,0326 \\
Tritoninfa & 1,5044 & 0,0703 & 1,2167 & 0,0714 \\
Fêmea & 1,79 & 0,0767 & 1,5207 & 0,057 \\
Macho & 1,9173 & 0,1065 & 1,6809 & 0,0441 \\
\hline
\end{tabular}

Tabela 1. Medidas do comprimento da carapaça e do fêmur III dos estágios de desenvolvimento de Cryptocellus simonis na serapilheira do Bosque Rodrigues Alves, Belém, Pará, Brasil, coletados entre março-novembro de 2001.

\begin{tabular}{ccccc}
\hline $\begin{array}{c}\text { Estágios de } \\
\text { desenvolvimento }\end{array}$ & Triagem Manual & $\begin{array}{c}\text { Técnicas de coleta } \\
\text { Extrator de } \\
\text { Winkler }\end{array}$ & $\begin{array}{c}\text { Funil de Berlese- } \\
\text { Tullgren }\end{array}$ & Total \\
\hline Larvas & 2 & 20 & 2 & 24 \\
Protoninfa & 7 & 15 & 3 & 25 \\
Deutoninfa & 3 & 11 & 4 & 18 \\
Tritoninfa & 6 & 10 & 2 & 18 \\
Macho & 4 & 4 & 3 & 11 \\
Fêmea & 5 & 10 & 0 & 15 \\
Total & 27 & 70 & 14 & 111 \\
Total \% & $24,32 \%$ & $63,06 \%$ & $12,61 \%$ & $100 \%$ \\
\hline
\end{tabular}

Tabela 2. Número de indivíduos de cada estágio de desenvolvimento de Cryptocellus simonis por técnica de coleta, na serapilheira do Bosque Rodrigues Alves, Belém, Pará, Brasil coletados entre março-novembro de 2001. 


\subsection{Amostragem}

Foram realizadas duas campanhas de coleta, a primeira entre os dias 26.03 e 12.05.2001 (período chuvoso) e a segunda entre 27.09 a 08.11.2001 (período seco). Durante a campanha do período chuvoso foram realizadas seis expedições à área de estudo, sendo obtidas 99 amostras (24 na primeira expedição e 15 em cada uma das restantes), 33 destinadas a cada uma das três técnicas de coleta. Durante a campanha do período seco foram realizadas quatro expedições, sendo obtidas 60 amostras, 15 em cada expedição, divididas igualmente entre as três técnicas. Ao término das duas campanhas, foram obtidas 159 amostras, das quais 53 foram destinadas a cada uma das três técnicas de coleta. Todas as amostragens ocorreram no período da manhã. Em cada expedição foi escolhido um canteiro, onde foram sorteados oito pontos na primeira expedição e cinco nas restantes. Foram escolhidos canteiros com menor grau de perturbação e com tamanho mínimo de $300 \mathrm{~m}^{2}$. Em cada ponto sorteado foram demarcados, com auxílio de balizas, três amostras de $1 \mathrm{~m}^{2}$ de área no solo, contíguas, a fim de minimizar diferenças na distribuição espacial das populações (efeito de agregação). Um novo sorteio foi organizado para selecionar a amostra que seria destinada a cada uma das três técnicas de coleta. Toda a serapilheira, bem como a camada mais profunda de material orgânico solto, presente em cada metro quadrado delimitado, foi colocada em um concentrador na forma de uma peneira de mão, de malha de $5 \mathrm{~mm}$ (figura 2). O material particulado resultante foi acondicionado em sacos plásticos lacrados. Todas as amostras foram levadas ao laboratório e encaminhadas para as respectivas técnicas de coleta.

\subsection{Técnicas de coleta}

Triagem manual de serapilheira: esta técnica consiste na colocação do material particulado resultante da amostragem em uma bandeja de plástico de $30 \mathrm{~cm}$ de largura por $50 \mathrm{~cm}$ de comprimento (figura 3), para permitir a triagem dos espécimes com o auxílio de pinças e pincéis. A bandeja utilizada permitiu a triagem de até 500 g de material particulado por rodada. Cada rodada de triagem teve a duração máxima de 25 min. Este procedimento foi executado no mesmo dia da amostragem.

Funil de Berlese-Tullgren: esta técnica consiste no acondicionamento do material particulado em funis metálicos de $20 \mathrm{~cm}$ de altura, $12 \mathrm{~cm}$ de diâmetro na circunferência superior e $3 \mathrm{~cm}$ de diâmetro na inferior. A 10 cm da superfície superior posiciona-se uma placa metálica perfurada para permitir a passagem dos espécimes. Os furos têm, em média, 5 mm de diâmetro. Cada funil comporta, em média, 300 g de material particulado. Os funis cheios foram colocados em uma caixa de contenção de madeira que acomoda nove funis. Na parte superior da caixa estão instaladas quatro lâmpadas de $50 \mathrm{~W}$ cada, que proporcionam a iluminação e o aquecimento da caixa (figura 4). A porta da caixa possui uma abertura gradeada que permite a ventilação, evitando o superaquecimento interno. Na parte inferior do funil foi posicionado um pote de plástico contendo álcool 80\%. Modelos similares a este foram utilizados por Bandeira \& Torres (1985). O número de funis utilizados em cada amostra variou de um a doze. O material particulado foi mantido no funil de Berlese-Tullgren durante sete dias.

Extrator de Winkler: esta técnica consiste na utilização de uma rede de contenção de tecido perfurado, de $40 \mathrm{~cm}$ de comprimento por $20 \mathrm{~cm}$ de largura, com malha de $4 \mathrm{~mm}$. Cada rede acomoda cerca de $600 \mathrm{~g}$ de material particulado. A rede cheia de material é colocada dentro de uma armação de metal, revestida por tecido resistente. A parte superior do extrator é vedada e pendurada por uma corda. Na parte inferior do extrator acopla-se um pote de plástico com álcool 80\% (figura 5). Modelos similares a este foram descritos como "miniWinklers” em Bestelmeyer et al. (2000). O número de extratores utilizados em cada amostra variou de um a três. As armadilhas ficaram armadas por sete dias.

\subsection{Análise dos dados}

Dados de pluviosidade média mensal do período de coleta na região metropolitana de Belém foram cedidos pela EMBRAPA/CPATU. Todas as amostras foram triadas em laboratório com auxílio de um estereomicroscópio Zeiss Stemi SV11. Animais de outras Ordens foram separados e acondicionados de forma a manter as informações de esforço amostral, para estudos posteriores. Para as análises estatísticas foram utilizados os programas BioEstat 3.0 (Ayres et al. 2003) e SYSTAT 10.2 (Wilkinson 1990). A diferença de abundância por técnicas foi testada através do Teste de Kruskal-Wallis (Teste $\mathrm{H}$ ). A diferença de abundância entre os períodos seco e chuvoso foi testada através do Teste de Mann-Whitney (Teste U). A relação entre os comprimentos da carapaça e do fêmur III foi testada através de Correlação Linear de Pearson enquanto que a relação entre a abundância e pluviosidade foi testada através de Regressão Linear Simples.

\section{RESULTADOS E DISCUSSÃO}

Obteve-se um total de 111 indivíduos de Cryptocellus simonis, sendo 26 adultos (11 machos e 15 fêmeas), e 85 jovens (61 ninfas e 24 larvas) (figura 6). A densidade média foi de 0,7 ind. $/ \mathrm{m}^{2}$, enquanto que a máxima foi de 18 ind./ $\mathrm{m}^{2}$. Platnick \& Pass (1982) estudaram uma população de Pseudocellus seacus Platnick \& Pass em uma floresta de transição na Guatemala Central, amostrada através de triagem manual, e encontraram valores médios de 0,4 ind. $/ \mathrm{m}^{2}$. Estes valores são similares aos encontrados no presente estudo. Entretanto, Adis et al. (1989) encontraram densidade muito mais alta em 
duas espécies simpátricas na Amazônia Central, Cryptocellus becki (36 ind. $\left./ \mathrm{m}^{2}\right)$ e C. adisi (10 ind./ $\left.\mathrm{m}^{2}\right)$ em áreas secundárias de terra firme (capoeira), apesar destes valores terem diminuído em áreas de floresta primária (C. becki: 7 ind. $/ \mathrm{m}^{2}$ e C. adisi: 5 ind. $/ \mathrm{m}^{2}$ ). Estes dados indicam que $C$. simonis pode apresentar padrões de abundância bastante diferentes dos relatados para $C$. becki e C. adisi. Entretanto, Legg (1977) registrou na África até 150 ind. $/ \mathrm{m}^{2}$.

Nos períodos chuvosos (março a maio) e seco (setembro a novembro) foram coletados, respectivamente, $78 \%$ e $75 \%$ de imaturos. Larvas foram mais abundantes no período seco, enquanto que protoninfas foram mais abundantes no período chuvoso (figura 7). O mês mais chuvoso foi o de abril (473 mm) enquanto que o mais seco foi o de novembro (106 mm). Não foi encontrada uma relação significativa $(\mathrm{p}>0,05)$ entre os dados de pluviosidade e de abundância de Ricinulei $\left(\mathrm{n}=10\right.$, g.l. $=1, \mathrm{R}^{2}=0,058, \mathrm{p}=$ 0,503). Também não foram encontradas diferenças na abundância de $C$. simonis ( $\mathrm{p}>0,05)$ quando os dados foram agrupados em duas categorias (períodos seco e chuvoso), de acordo com os dados de pluviosidade (Mann-Whitney, $\mathrm{n}=53$, g.l. = 1, U = 333,5, p = 0,946). Adis et al. (1989) também não encontraram correlação entre os dados climáticos e a abundância C. becki e C. adisi na Amazônia Central.

A presença de larvas de $C$. simonis foi registrada em ambos os períodos (oito no chuvoso e 16 no seco) indicando que não existe um período reprodutivo definido, fato também observado em C. becki por Adis et al. (1989), a espécie dominante na região de Manaus. A iteroparidade pode representar uma estratégia para manutenção da população, pois os Ricinulei produzem um único ovo por vez e a presença de espermateca para armazenar espermatozóides pode garantir mais de uma fecundação com a mesma cópula (Legg 1977).

As medidas de carapaça e fêmur III para cada estágio de desenvolvimento podem ser visualizadas na tabela 1 . Foi encontrada uma correlação positiva significativa entre o comprimento da carapaça e o comprimento do fêmur III (Correlação Linear de Pearson, $n=111$, g.l. $=109$, $r=0,9874$, $\mathrm{p}$ $=0,00001$ ) indicando grandes diferenças de tamanho para cada estágio de desenvolvimento de C. simonis (figura 8). As medidas de carapaça de machos e fêmeas conferem com as medidas de Platnick \& Shadab (1977), feitas com base em material coletado na mata do Utinga, também na região metropolitana de Belém. No entanto, espécimes do Bosque Rodrigues Alves apresentam o comprimento do fêmur III bem maior que o relatado para os espécimes do Utinga. Isto poderia indicar a ocorrência de diferenças morfométrica consistentes entre estas duas populações, atualmente isoladas pela paisagem urbana. Os dados apresentados neste trabalho corroboram os obtidos por Adis et al. (1989) que também encontraram grandes diferenças de tamanho por estágio de desenvolvimento de $C$. becki, utilizando a relação entre o comprimento da carapaça e comprimento total do corpo.
A abundância em cada estágio de desenvolvimento, obtida em cada técnica de coleta, e a eficiência das três técnicas podem ser visualizado na tabela 2 . Foram encontradas diferenças significativas $(p<0,05)$ na abundância de $C$. simonis entre as técnicas de coleta (Kruskal-Wallis, $\mathrm{n}=159$, g.l. $=2, \mathrm{H}=7,297$, $\mathrm{p}=0$,026). O Teste de Comparação Múltipla de Dunn apontou diferenças significativas $(p>0,05)$ apenas entre o extrator de Winkler e os funis de Berlese-Tullgren, indicando a baixa eficiência dos funis em relação aos extratores e a equivalência da triagem manual em relação tanto aos extratores quanto aos funis. A utilização de apenas uma técnica de coleta pode explicar, em parte, a aparente ausência de Ricinulei em outros levantamentos realizados na Amazônia Oriental (Pará), onde foram realizadas coletas intensivas de fauna de solo, com o uso de Funis de Berlese-Tullgren similares aos usados no presente trabalho (Bandeira \& Torres 1985, 1988, Macambira 1997). A grande maioria das espécies é conhecida por poucos exemplares e Cooke (1967) suspeitava que esta aparente raridade estava muito mais relacionada com o método de amostragem do que com a abundância real do grupo.

A escolha da técnica de coleta mais adequada para o inventário de Ricinulei deve levar em conta, além da eficiência de captura, também a praticidade da sua utilização em campo. Durante o procedimento de triagem manual, foi possível observar o comportamento de tanatose em alguns indivíduos, tanto jovens quanto adultos. Este comportamento pode ter condicionado o baixo valor obtido por esta técnica (24,32\%) em relação ao extrator de Winkler. Os funis de Berlese-Tullgren, além de terem sido responsáveis pela captura de apenas 12,61\%, necessitam de energia elétrica e o seu transporte ao campo é inviável. Por outro lado, tanto os extratores de Winkler quanto a triagem manual podem ser utilizados durante as expedições. A desvantagem da triagem manual em relação ao extrator de Winkler é o grande despêndio de tempo necessário para implementá-la. O extrator de Winkler foi responsável pela captura de 63\% do total de indivíduos e provou ser a melhor técnica, não só pela praticidade como também pela eficiência, na captura de Ricinulei em inventários faunísticos na Amazônia Oriental.

\section{AGRADECIMENTOS}

As Coodenadorias de Fauna e Flora do Bosque Rodrigues Alves pelo apoio logístico; a The Nature Conservancy (TNC) pelo apoio financeiro no IV Encontro de Aracnólogos do Cone Sul (JAPB); ao Dr. Juarez Pezzuti (NAEA-UFPA) e a Dra. Ana Albernaz (CCTE-MPEG) pela ajuda nas análises dos dados; a dois revisores anônimos pelas sugestões. Ao CNPq, pelo apoio financeiro (RPR/ABB). 


\section{REFERÊNCIAS BIBLIOGRÁFICAS}

ADIS, J. \& HARVEY, M.S. 2000. How many Arachnida and Myriapoda are there World-Wide and in Amazonia? Stud. Neotrop. Fauna Environ. Short Communication 35: 139-141.

ADIS, J.U., PLATNICK N.I., MORAIS, J.W.de \& RODRIGUES, J.M.G. 1989. On the abundance and ecology of Ricinulei (Arachnida) from Central Amazonia, Brazil. J. New York Ent. Soc. 97(2): 133-140.

AYRES, M., AYRES Jr., M., AYRES, D.M. \& SANTOS, A.S. 2003. BioEstat 3.0: aplicações estatísticas nas áreas das ciências biológicas e médicas. Belém: Sociedade Civil Mamirauá, Brasília CNPq. 290p.

BANDEIRA, A.G. \& TORRES, M.F.P. 1985. Abundância e distribuição de invertebrados do solo em ecossistemas da Amazônia Oriental. O papel ecológico dos cupins. Bolm Mus. Para. Emílio Goeldi, sér. Zool. 2(1): 13-38.

BANDEIRA, A.G. \& TORRES, M.F.P. 1988. Considerações sobre densidade, abundância e variedade de invertebrados terrestres em áreas florestais de Carajás, sudeste da Amazônia. Bolm Mus. Para. Emílio Goeldi, sér. Zool. 4(2): 191-200.

BESTELMEYER, B.T, AGOSTI, A., ALONSO, L.E., BRANDÃO, C.R.F., BROWN Jr., W.L., DELABIE, J.H.C. \& SILVESTRE, R. 2000. Field techniques for the study of ground-dwelling ants. In: (D. Agosti, J.D. Majer, L.E. Alonso \& T.R. Schultz (eds.). Ants: Standard Methods for Measuring and Monitoring Biodiversity (Smithsonian Institution Press, Washington and London, p.122-144.

BONALDO, A.B. \& PINTO-DA-ROCHA, R. 2003. On a new species of Cryptocellus from the Brasilian Amazon (Arachnida, Ricinulei). Revta Ibérica de Aracnol. 7: 103-108.

COOKE, J.A.L. 1967. Observations on the biology of Ricinulei (Arachnida) with description of two new sapecies of Cryptocellus. J. Zool. 151: 31-42.

HARVEY, M.S. 2002. The neglected cousins: what do we know about the smaller arachnid orders? J. Arachnology 30: 357-372.

LEGG, G. 1977. Sperm transfer and mating in Ricinoides hanseni (Ricinulei: Arachnida). J. Zool. 182: 51-61.

LEVI, H.W. 1964. Nineteenth century South American araneology. Papéis Dep. Zool. S. Paulo16: 9-19.

MACAMBIRA, M.L.J. 1997. A fauna de invertebrados do solo. In: P. Lisboa (org.). Caxiuanã. Museu Paraense Emílio Goeldi, Belém, Brasil, p. 355-360.

OVERAL, W. L. \& MASCARENHAS, B. M. 1993. Recomendações para o inventário faunístico da Amazônia. Bolm Mus. Para. Emílio Goeldi, sér. Zool. 9(2): 329-339.

PLATNICK, N.I. 1988. A new Cryptocellus (Arachnida, Ricinulei) from Brazil. J. New York Ent. Soc. 96(3): 363-366.
PLATNICK, N.I. 2002. Ricinulei. In Amazonian Arachnida and Myriapoda (J. ADIS, org.). Pensoft Publishes, Sofia, Moscow, p.381-386.

PLATNICK, N.I. \& PASS, G. 1982. On a new Guatemalan Pseudocellus (Arachnida, Ricinulei). Am. Mus. Novit. 2733: 1-6.

PLATNICK, N.I. \& PAZ, N.S. 1979. On the Cryptocellus magnus Group (Arachnida, Ricinulei). Am. Mus. Novit. 2677: 1-9.

PLATNICK, N.I. \& SHADAB, M.U. 1977. On Amazonian Cryptocellus (Arachnida, Ricinulei). Am. Mus. Novit. 2633: 1-58.

WILKINSON, L. 1990. SYSTAT: The System for Statistics. Systat Inc., Evanston, Illinois. 822p.

Título: Abundância e fenologia de Cryptocellus simonis Hansen \& Sorensen, 1904 (Ricinulei, Arachnida) na serapilheira do Bosque Rodrigues Alves, Belém, Pará, Brasil, com a comparação de três técnicas de coleta

Autores: José Augusto Pereira Barreiros, Ricardo Pintoda-Rocha \& Alexandre Bragio Bonaldo

Biota Neotropica, Vol. 5( número 1a): 2005 http://www.biotaneotropica.org.br/v5n1a/pt/ abstract?article+BN004051a2005

Recebido em 07/12/2003 - Aceito em 01/02/2005

ISSN 1676-0603 